\title{
Vitamin D Deficiency and Its Relationship with Child-Pugh Class in Patients with Chronic Liver Disease
}

\author{
Zubia Jamil*1 ${ }^{1}$, Sharmin Arif ${ }^{1}$, Anum Khan ${ }^{1}$, Asghar Aurangzeb Durrani ${ }^{2}$ and Nayyar Yaqoob ${ }^{2}$ \\ ${ }^{1}$ Department of Medicine, Foundation University Medical College, Islamabad, Pakistan; ${ }^{2}$ Department of Medicine, \\ Fauji Foundation Hospital, Rawalpindi, Pakistan
}

\begin{abstract}
Background and Aims: Skeletal manifestation in liver diseases represents the minimally scrutinized part of the disease spectrum. Vitamin D deficiency has a central role in developing hepatic osteodystrophy in patients with chronic liver disease. This study aimed to investigate vitamin $D$ levels and their relationship with disease advancement in these patients. Methods: Vitamin D levels were checked in 125 chronic liver disease patients. The patients were classified in three stages according to Child-Pugh score: A, B and C. The relationship of vitamin $D$ levels with Child-Pugh score and other variables in the study was assessed by the contingency coefficient. Correlation and logistic regression analyses were also carried out to find additional predictors of low vitamin D levels. Results: Among the patients, $88 \%$ had either insufficient or deficient stores of vitamin $D$, while only $12 \%$ had sufficient vitamin D levels $(p>0.05)$. Vitamin D levels were notably related to Child-Pugh class (contingency coefficient $=0.5, p<0.05$ ). On univariate and multinomial regression analyses, age, female sex, MELD and Child-Pugh class were predictors of low vitamin D levels. Age, model of end-stage liver disease score and Child-Pugh score were negatively correlated to vitamin $D$ levels $(p<0.05)$. Conclusions: Vitamin D deficiency is notably related to age, female sex and model of end-stage liver disease score, in addition to Child-Pugh class of liver cirrhosis. Vitamin D levels should be routinely checked in patients with advanced liver cirrhosis (Child-Pugh class B and C) and this deficiency must be addressed in a timely manner to improve general well-being of cirrhotic patients.
\end{abstract}

Citation of this article: Jamil Z, Arif S, Khan A, Durrani AA, Yaqoob N. Vitamin $D$ deficiency and its relationship with ChildPugh class in patients with chronic liver disease. J Clin Transl Hepatol 2018;6(2):135-140. doi: 10.14218/JCTH.2017.00055.

\section{Introduction}

Liver cirrhosis has exerted a major burden on public health globally, being the $4^{\text {th }}$ leading cause of death in many countries. ${ }^{1}$ It is estimated that around one million deaths are

Keywords: Vitamin D deficiency; Liver cirrhosis; Osteodystrophy; Liver disease; Skeletal manifestations.

Abbreviations: CLD, chronic liver disease; CP, Child-Pugh; PT, prothrombin time; INR, international normalized ratio.

Received: 13 August 2017; Revised: 20 October 2017; Accepted: 12 December 2017

* Correspondence to: Zubia Jamil, Department of Medicine, Foundation University Medical College, House No 98, Street No 18, Phase 6 Bahria Town, Islamabad 44000, Pakistan. Tel: +92-3225030457, E-mail: zubiajamil321@gmail.com attributed to liver cirrhosis each year. ${ }^{2}$ The foremost cause of liver cirrhosis in many countries of the world is chronic hepatitis $C$ virus infection. ${ }^{3}$ Many other etiological agents, such as chronic hepatitis B infection, nonalcoholic steatotic hepatitis, alcohol, cholestatic disorders, chronic vascular obstructive diseases and autoimmune diseases, also result in liver cirrhosis. ${ }^{1,3}$

The Child-Pugh (CP) score is a widely used scoring system to predict the 1-year survival rate among cirrhotic patients. First described by Child and Turcotte and later modified by Pugh et al. $^{4}{ }^{4}$ this score uses five parameters (hepatic encephalopathy, prothrombin time (PT), ascites, serum bilirubin and serum albumin) to classify the patients in early, intermediate and advanced stages of liver cirrhosis. Esophageal varices, ascites, portal hypertension, hepatic encephalopathy and hepatocellular carcinoma are all well studied and well-known complications of liver cirrhosis. ${ }^{5}$ A less studied complication of liver cirrhosis, however, is represented by its skeletal manifestations.

Liver cirrhosis forms a complex relationship between serum calcium, phosphate, parathyroid hormone and vitamin D levels, affecting the musculoskeletal system. These skeletal manifestations in liver disease are termed hepatic osteodystrophy. Hepatic osteodystrophy results in a high risk of fractures in cirrhotic patients, thus negatively affecting their morbidity. ${ }^{6,7}$ Vitamin $D$ deficiency plays a key role in development of hepatic osteodystrophy. ${ }^{7}$ Fish, eggs and oral supplements are dietary sources of vitamin D (fat soluble vitamin); however, the major source is sun exposure of the skin. Pro-vitamin $D_{3}$ (cholecalciferol), whether obtained from diet or sunlight, remains stored in fat cells until the first step of hydroxylation takes place in the liver, resulting in the synthesis of 25-hydroxy cholecalciferol. 1,25 -dihydroxy cholecalciferol (active vitamin D) is synthesized in the kidneys by the final step of hydroxylation. ${ }^{8}$

Vitamin $D$ is necessary for functions and health of both muscles and bones in our body. In addition, vitamin D has many extra skeletal functions. Many chronic diseases, like diabetes mellitus, chronic infections and malignancy (breast, prostate and colon), are less likely to develop with presence of adequate stores of vitamin $D$ in our body because many cells of the body like hepatocytes, macrophages, immune $B$ and Tcells express vitamin D receptors on their surface. ${ }^{9,10}$ Patients suffering from chronic liver diseases are mostly vitamin $D$ deficient. It is estimated about one-third of cirrhotic patients have vitamin D deficiency. ${ }^{6}$

There are multiple mechanisms by which vitamin $D$ deficiency occurs in these patients.

1. Cholestasis due to CLD results in malabsorption of fats and fat soluble vitamins $(A, D, E, K){ }^{11}$ 
2. Anorexia due to chronic illness resulting in dietary deficiency of vitamin D. ${ }^{11}$

3. Reduced mobility due to chronic illness resulting in limited sun exposure and reduced levels of cholecalciferol. ${ }^{12}$

4. Inhibition of hydroxylation of cholecalciferol in liver diseases results in a reduced synthesis of 25-hydroxy cholecalciferol. ${ }^{13}$

Skeletal manifestations of CLD are a neglected part of the disease spectrum. Yet, they significantly affect the morbidity of patients with liver diseases. The primary aim of this study was to document vitamin $D$ deficiency in patients with liver cirrhosis and to find its relationship with CP class of the liver cirrhosis. Studying the other factors which lead to vitamin $D$ deficiency in these patients was an additional aim of this study.

\section{Methods}

A cohort study was conducted in Fauji Foundation Hospital, Rawalpindi, Pakistan from January 2016 to July 2016. Patients were labeled as a case of CLD if they had any one, or more than one, of the following features: biochemical abnormalities suggesting CLD, like deranged liver function tests in the presence of risk factors leading to CLD (duration >3 months); ultrasonographic findings of CLD (surface nodularity, coarse and heterogeneous liver texture and segmental hypertrophy or atrophy) $i^{14}$ chronic liver parenchymal changes on liver biopsy; medical records suggesting CLD (medications or previous admissions due to hepatic encephalopathy, ascites or variceal bleeding).

Ultimately, 125 patients fulfilling these criteria were selected for this study. Patients had been excluded from the study if: taking medications which can affect the serum vitamin D levels, like vitamin A, calcium and vitamin D supplements, steroids, antiepileptic drugs and bisphosphonates, etc.; suffering from chronic diseases that can impair calcium and vitamin $D$ metabolism, like chronic kidney disease, malabsorption syndrome, tuberculosis, etc.; presence of hepatocellular carcinomas or other malignancies.

Ethical approval was given by the hospital prior to the study's commencement. Informed consent was obtained from the participant patients. A complete demographic profile of patients was noted. Detailed history and complete examination of patients was carried out. The blood samples of patients were taken and sent for complete blood examination, and testing for levels of serum bilirubin, serum albumin, the international normalization ratio (INR) and 25-hydroxyvitamin D. Hepatitis serology for hepatitis C and hepatitis B were also carried out to find the cause of CLD.

Patients were scored according to Child-Pugh classification and classified into three classes: A, B and C. Five parameters were used to give these scores to patients. The parameters were ascites, hepatic encephalopathy, serum bilirubin $(\mathrm{mg} / \mathrm{dL})$, INR and serum albumin $(\mathrm{g} / \mathrm{dL})$. Each parameter was scored 1,2 or 3 . The minimum score of CP class was 5 and the maximum was 15 . Ascites was graded as mild, moderate or severe by performing a transabdominal ultrasound (Famio 5 Ultrasound Machine; Abex Medical System, Toshiba, Japan). Grades of encephalopathy were determined by a physician during examination. Blood $(3 \mathrm{~mL})$ was taken in serum bottles for serum albumin and serum bilirubin, and results were obtained by using a chemistry analyzer (Dimensions RxL Max Chemistry Analyzer; Siemens Healthineers Laboratories, USA). Blood ( $3 \mathrm{~mL}$ in a tube containing 3.2\% Na-citrate) was used to determine PT, and results were obtained by using the Sysmex CA-500 Coagulation Analyzer (Siemens Healthcare Diagnostics, Japan). INR was calculated manually by using the patient and control PTs.

Those patients without ascites or hepatic encephalopathy, serum bilirubin $<2 \mathrm{mg} / \mathrm{dL}$, serum albumin $>3.5 \mathrm{~g} / \mathrm{dL}$ and INR $<1.7$ were scored as 5 and 6 , and they were classified as CP class A. Patients with mild to moderate ascites, grade 1 or grade 2 hepatic encephalopathy, serum bilirubin $2-3 \mathrm{mg} / \mathrm{dL}$, serum albumin $2.8-3.5 \mathrm{~g} / \mathrm{dL}$ and INR $1.7-2.3$ were given a score from 7 to 9 , and they were classified as CP class B. Those patients who had massive ascites and grade 3 or grade 4 hepatic encephalopathy, serum bilirubin $>3 \mathrm{mg} / \mathrm{dL}$, serum albumin $<2.5 \mathrm{~g} / \mathrm{dL}$ and INR $>2.3$ were placed in $\mathrm{CP}$ class $\mathrm{C}$, as the scores of their parameters were from 10 to 15 .

Blood ( $2 \mathrm{~mL}$ ) was drawn from patients to check vitamin $D$ (25-hydroxyvitamin D) levels. Levels were checked in serum using the Elecsays Analyzer (Cobas_e_411 Analyzer; Roche Diagnostics, Germany). Deficiency was defined as vitamin D levels $<20 \mathrm{ng} / \mathrm{mL}$; between $21 \mathrm{ng} / \mathrm{mL}$ to $30 \mathrm{ng} / \mathrm{mL}$ was considered insufficient vitamin $D$ and above $31 \mathrm{ng} / \mathrm{mL}$ was considered sufficient. The data of 125 patients with CLD were collected. In addition, 100 healthy individuals were taken as controls, being age- and sex-matched to the disease group.

The mean of vitamin D levels in both groups (patients and controls) was tested by student's $t$-test. Quantitative variables were expressed in terms of mean and standard deviation. Frequency and percentage were used for qualitative measures. The $p$-value was calculated by the contingency coefficient to find a relationship of vitamin $D$ levels to $C P$ scores of liver cirrhosis and also with other variables. Both correlation and logistic regression analyses were also carried out to find additional predictors of vitamin D deficiency in the disease group. SPSS version 20 software (USA) was used for analyzing the data.

\section{Results}

Most of the patients in the study were females, as the study was conducted in an ex-servicemen beneficiary hospital. Among the 125 patient participants, 115 (92\%) were females and only $10(8 \%)$ were males. The minimum age of the patients was 18 years and the maximum was 84 years, with mean age of 56.88 years.

Among the 125 patients, 117 (93.6\%) were affected by chronic hepatitis $C$ infection. The second cause of CLD was hepatitis B infection, affecting $6(4.8 \%)$ patients; the remaining $2(1.6 \%)$ patients had seronegative liver disease. Patients were categorized according to $\mathrm{CP}$ class to assess the degree of liver dysfunction. Among the 125 patients, 38 (30.4\%) had advanced liver disease and were in CP class C, $48(38.4 \%)$ were CP class A, showing early liver cirrhosis, and 39 (31.2\%) were $\mathrm{CP}$ class $\mathrm{B}$, indicating the intermediate stage of liver cirrhosis.

The distribution of some baseline variables among the study group is shown in Table 1.

The results of the study showed that many patients suffering from cirrhosis of liver had either deficient or insufficient vitamin $D$ levels. Vitamin $D$ levels in these patients ( $n=125$ ) were lower than vitamin $\mathrm{D}$ levels in the control group, ranging from $23.16 \pm 7.94 \mathrm{ng} / \mathrm{mL}$ to $6.00-44.00 \mathrm{ng} / \mathrm{mL}$ and $34.13 \pm 18.34 \mathrm{ng} / \mathrm{mL}$ to $9.00-87.00 \mathrm{ng} / \mathrm{mL}$ respectively. Although, statistically, this difference was not significant $(p>0.05)$. Patients were categorized into three groups 
Jamil Z. et al: Vitamin D deficiency in chronic liver disease

Table 1. Distribution of variables among the 125 patients with chronic liver disease

\begin{tabular}{llllll}
\hline & $n$ & Minimum & Maximum & Mean & Standard deviation \\
\hline Hemoglobin, g/dL & 125 & 7.80 & 15.60 & 9.43 & 2.15 \\
WCC $\times 10^{9}$ cells/L & 125 & 1.72 & 20.20 & 6.22 & 3.15 \\
Platelets $\times 10^{9} / \mathrm{L}$ & 125 & 24.00 & 475.00 & 112.86 & 75.64 \\
Bilirubin, $\mathrm{mg} / \mathrm{dL}$ & 125 & 0.41 & 26.02 & 2.24 & 2.01 \\
ALT, U/L & 125 & 20.00 & 302.00 & 55.53 & 38.37 \\
ALP, U/L & 125 & 102.00 & 575.00 & 218.50 & 88.68 \\
INR & 125 & 1.00 & 2.60 & 1.67 & 0.46 \\
Albumin, g/dL & 125 & 2.20 & 4.30 & 3.53 & 0.44 \\
CP score & 125 & 5.00 & 13.00 & 7.90 & 2.27 \\
MELD score & 125 & 10.00 & 32.00 & 19.56 & 6.38 \\
\hline
\end{tabular}

Abbreviations: ALP, alkaline phosphatase; ALT, alanine aminotransferase; CP, Child-Pugh; INR, international normalized ratio; MELD, model of end-stage liver disease; WCC, white cell count.

according to vitamin $D$ levels: patients with deficient $(<20 \mathrm{ng} / \mathrm{mL})$, insufficient $(20-30 \mathrm{ng} / \mathrm{mL})$ and sufficient $(>30 \mathrm{ng} / \mathrm{mL})$ Vitamin D levels. The majority of cirrhotic patients $(n=66)$ had insufficient vitamin D levels (patients: $52.8 \%$ vs. controls: $27 \%$ ). Vitamin D deficiency was also common in these cirrhotic patients. Forty-three patients had deficient levels (patients: $34.4 \%$ vs. controls: $26 \%$ ). Sufficient levels of vitamin D were found in only 16 patients with liver cirrhosis (patients: $12.8 \%$ vs. controls: $47 \%$ ). The patients and controls groups are illustrated in a bar chart according to these three categories of vitamin D (Fig. 1).

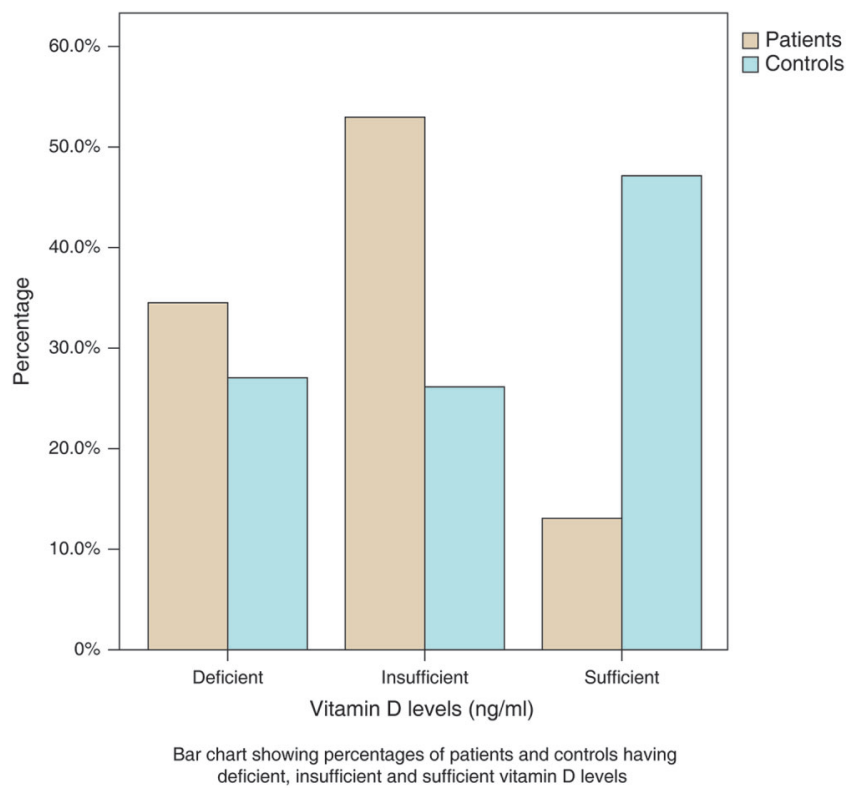

Fig. 1. Patients with chronic liver disease and controls according to vitamin D categories. The $X$-axis shows the three categories of vitamin $D$ levels: deficiency $(<20 \mathrm{ng} / \mathrm{mL})$, insufficiency $(20-30 \mathrm{ng} / \mathrm{mL})$ and sufficiency $(>30 \mathrm{ng} / \mathrm{mL})$. The $\mathrm{Y}$-axis shows the frequency of patients and controls according to the three categories of vitamin $\mathrm{D}$. The majority of cirrhotic patients $(52.8 \%, n=66$; controls: $27 \%$ ) had insufficient vitamin D levels. Vitamin D deficiency was found in $34.4 \%$ ( $n=43$; controls: $26 \%$ ). Sufficient levels of vitamin D were found in only $12.8 \%$ ( $n=16$; controls: $47 \%)$ of patients with liver cirrhosis.
The primary aim of this study was to find the association of vitamin D levels with CP class of liver cirrhosis. Although vitamin D levels were not statistically lower than the control group, but vitamin $\mathrm{D}$ levels were strongly related to $\mathrm{CP}$ classification of liver cirrhosis. Most patients with CP class $C$ were vitamin $D$-deficient, while none had sufficient vitamin $D$ stores. In contrast to this, 12 patients having CP class A had sufficient vitamin $D$ levels. The results indicated that vitamin $D$ levels in cirrhotic patients are associated with CP classification ( $p<0.05$ and contingency coefficient $=0.5$ ) (Table 2 ). Thus, as the CP class advances, vitamin D levels decrease (Fig. 2).

The association of vitamin D levels was also studied with different variables, as the secondary aim of the study. The three groups of vitamin $D$ levels showed significant association with age of patients $(p=0.000)$, serum albumin $(p=$ $0.045)$, model of end-stage liver disease score $(p=0.003)$ and CP score $(p=0.000)$, in addition to the CP class of liver cirrhosis. The association of three categories (deficient, insufficient and sufficient) vitamin D levels with various variables is shown in Table 3. Both regression analysis and correlation analysis were carried out to find the predictors of vitamin D deficiency. In univariate analysis, age, female sex, model for end-stage liver disease score, CP score and CP class $(p<0.05)$ were found to be predictors of vitamin D deficiency.

Multinomial analysis was also carried out. The model-fitting information showed that the model was statistically fit, with chi-square test value of 90.30 and $p$ value of 0.000 . The model covered $54 \%$ to $63 \%$ variation of variables (Cox and Snell pseudo $R^{2}$ and Nagelkerke pseudo $R^{2}$ respectively) and classified $93.5 \%$ of cases. Among quantitative variables age, model for end-stage liver disease score and CP score were found to be predictors of vitamin D deficiency. Female sex and CP class were found to be predictors of low vitamin D levels among qualitative variables. Etiology of liver cirrhosis was not found to be a predictor of low vitamin D levels in our study. Both univariate and multinomial regression analyses of different variables to predict vitamin D deficiency among study group are summarized in Table 4.

Pearson correlation was also carried out to find the relationship of all variables with vitamin D levels. Significant correlation of vitamin $D$ levels was found with age (correlation coefficient $=-0.594, p=0.000$ ), serum albumin (correlation 
Jamil Z. et al: Vitamin D deficiency in chronic liver disease

Table 2. Relationship of vitamin D levels with CP class of cirrhosis of the liver

\begin{tabular}{|c|c|c|c|c|}
\hline \multirow[b]{2}{*}{ Categories of vitamin D } & \multicolumn{4}{|l|}{ CP class } \\
\hline & $A$ & B & $\mathrm{C}$ & $n$ \\
\hline Deficient & $7(14.5 \%)$ & $7(17.9 \%)$ & $29(76.3 \%)$ & $43(34.4 \%)$ \\
\hline Insufficient & $29(60.4 \%)$ & $28(71.7 \%)$ & $9(23.6 \%)$ & $66(52.8 \%)$ \\
\hline Sufficient & $12(25 \%)$ & $4(10.2 \%)$ & 0 & $16(12.8 \%)$ \\
\hline$n$ & 48 & 39 & 38 & 125 \\
\hline
\end{tabular}

Abbreviation: $\mathrm{CP}$, Child-Pugh.

coefficient $=0.297, p=0.001)$, model for end-stage liver disease score (correlation coefficient $=-0.452, p=0.000$ ) and CP score (correlation coefficient $=-0.556, p=0.000$ ).

\section{Discussion}

One-third of patients with liver cirrhosis suffer from vitamin D deficiency, and it results in hepatic osteodystrophy in these patients. ${ }^{6,15}$ This study aimed to document vitamin D deficiency in CLD patients. Although the cause of vitamin D in cirrhotic patients is multifactorial, the main mechanism through which cirrhosis of the liver causes vitamin D deficiency is the inhibition of vitamin $D$ hydroxylation. Mean vitamin $D$ levels were $23.16 \mathrm{ng} / \mathrm{mL}$ (controls: $34.14 \mathrm{ng} / \mathrm{mL}$ ) in CLD patients according to this study, indicating that many patients with CLD are suffering from insufficient stores of vitamin $D$, which can be responsible for many musculoskeletal manifestations. Deficient vitamin D stores ( $<20 \mathrm{ng} / \mathrm{mL}$ ) were found in $34 \%$ of patients. A large number of cirrhotic patients had either deficient or insufficient vitamin D levels; meanwhile, sufficient stores of vitamin $D(>30 \mathrm{ng} / \mathrm{mL})$ were found only in $12 \%$ of cirrhotic patients.

Many comprehensive studies and trials have been conducted to document vitamin D deficiency among cirrhotic patients. Zhao et al. ${ }^{15}$ conducted a study on 345 cirrhotic

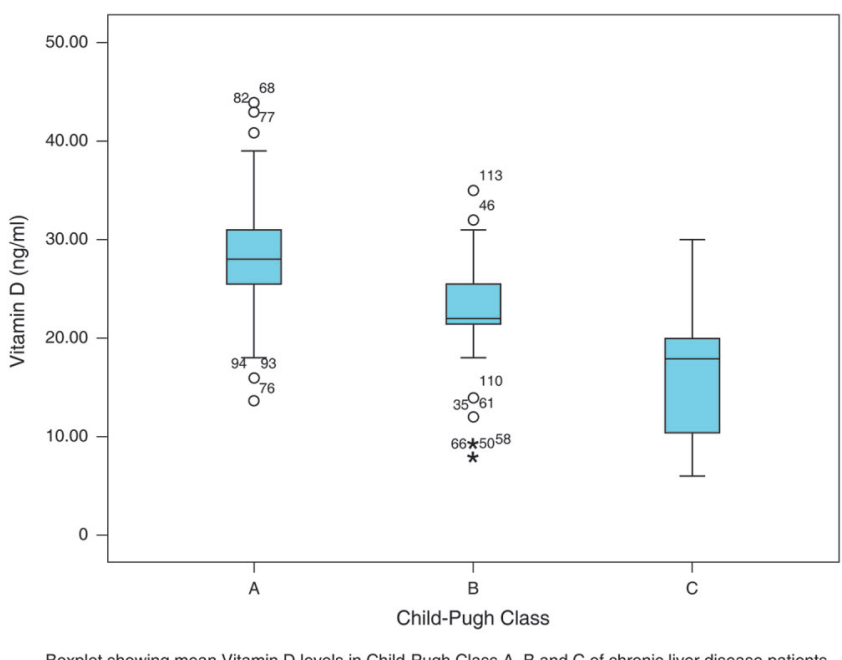

Fig. 2. Vitamin $D$ levels in Child-Pugh class $A$, B and $C$ for patients with chronic liver disease. Vitamin $D$ levels were much lower in class $C$, as compared to classes A and B. Vitamin D levels gradually decreased from class A to class C, meaning that as the disease advances the levels decrease further. patients and found that vitamin D levels were significantly deficient in these patients. One more study conducted in Spain by Fernandez et al. ${ }^{12}$ found that among 94 cirrhotic patients $87 \%$ had deficient levels of vitamin D. Kumar et al. ${ }^{16}$ conducted a study on 160 cirrhotic patients and found inadequate levels of vitamin $D$ in $80 \%$ of patients. We found that only $12 \%$ of patients had sufficient vitamin D levels, while $88 \%$ of patients do not have enough stores of vitamin $D$. These collective studies concur with our results, in that vitamin $D$ is deficient in patients suffering from CLD.

The mean vitamin $D$ levels in patients were lower than mean vitamin $D$ levels in the control group, although not significant statistically $(p>0.05)$. The reason is that a deficiency of vitamin $D$ is documented even in the healthy population of many countries. One study conducted by Kiani et al. ${ }^{17}$ found that $83 \%$ patients presenting in the outpatient department with different diseases had deficient levels of vitamin $D$, with mean levels of $13 \mathrm{ng} / \mathrm{dL}$. One more study conducted by Mehboobali et al. ${ }^{18}$ on 858 subjects found that $55 \%$ of healthy individuals in third world countries have vitamin $D$ deficiency. The causes of vitamin D deficiency in these healthy populations are multifactorial, including dietary, environmental and genetic causes. ${ }^{17,18}$ If vitamin D deficiency is already common in such healthy individuals, then how much does cirrhosis of the liver play a role in causing further vitamin $D$ deficiency in such patients belonging to those populations? This question needs to be further studied.

The primary aim was to study the relationship of vitamin $D$ levels with CP class of liver cirrhosis. We found that vitamin $D$ levels were negatively correlated with both model of end-stage liver disease score and CP score, suggesting that as the disease advances vitamin $D$ levels become more deficient $(p<0.05)$. The results of the study are similar to many other studies which have shown that vitamin $D$ level is inversely correlated to the CP score and model of end-stage liver disease score. ${ }^{19}$ Two studies conducted by Fernandez et al. and Zhao et al. ${ }^{12,15}$ both found that vitamin $D$ decreases further as cirrhosis of liver advances. Thus, patients with higher scores in the CP classification and model of end-stage liver disease score have notably lower vitamin D levels compared to patients with lower CP and model of end-stage liver disease scores.

Additional predictors of low vitamin D levels were also studied as the secondary aim of this study. Increasing age and female sex were found to be predictors of low vitamin $D$ levels $(p=0.000)$, in addition to CP class. Junaid et al. ${ }^{20}$ studied the main reasons of vitamin $D$ deficiency in a healthy population and found that the lack of education regarding a proper fortified diet, limited sun exposure $(<30 \mathrm{~m})$ and insufficient use of calcium and vitamin D 
Jamil Z. et al: Vitamin D deficiency in chronic liver disease

Table 3. Distribution of variables among patients with deficient, insufficient and sufficient vitamin $D$ levels

\begin{tabular}{|c|c|c|c|c|}
\hline Variable & Deficient, $n=43$ & Insufficient, $n=66$ & Sufficient, $n=16$ & $p^{*}$ \\
\hline Age in years & 67.71 & 51.98 & 49.23 & 0.000 \\
\hline Hemoglobin, g/dL & 9.64 & 9.28 & 9.48 & 0.710 \\
\hline WCC $\times 10^{3}$ cells $/ L$ & 6.33 & 6.08 & 6.47 & 0.866 \\
\hline Platelets $\times 10^{3}$ cells $/ \mathrm{L}$ & 94.35 & 118.47 & 137.11 & 0.099 \\
\hline PT, sec & 6.02 & 3.69 & 2.94 & 0.076 \\
\hline INR, sec & 1.90 & 1.60 & 1.40 & 0.178 \\
\hline Bilirubin, mg/dL & 3.03 & 1.99 & 1.30 & 0.268 \\
\hline$A L T, U / L$ & 52.73 & 54.77 & 65.41 & 0.506 \\
\hline$A L P, U / L$ & 228.69 & 217.15 & 198.58 & 0.494 \\
\hline Albumin, g/L & 3.42 & 3.57 & 3.65 & 0.045 \\
\hline MELD score & 23.19 & 18.43 & 14.94 & 0.003 \\
\hline CP score & 9.40 & 7.33 & 6.41 & 0.000 \\
\hline
\end{tabular}

* Bold font indicates statistically significant values.

Abbreviations: ALP, alkaline phosphatase; ALT, alanine aminotransferase; CP, Child-Pugh; INR, international normalized ratio; MELD, model of end-stage liver disease; WCC, white cell count.

supplements in daily life are the main causes of vitamin D deficiency. Female sex, low socioeconomic status, urban residency, smoking and polymorphisms in the sequences encoding vitamin $\mathrm{D}$ binding proteins are additional studied risk factors, which contribute to deficiency of vitamin $D$ in the healthy populations of these countries.

We found negative correlation between age and CP scores. As the age and CP score increases, the levels further decrease. These results are very much similar to the results of the study conducted by Kumar et al., ${ }^{16}$ who also found negative correlation of age and CP scores with vitamin D levels. They did not, however, find any association of vitamin $D$ levels with the etiology of liver diseases. In our study, we also did not find the etiology of liver cirrhosis to be as a predictor of low vitamin D levels.

Vitamin D deficiency is not only responsible for musculoskeletal manifestations but also many other complications in cirrhotic patients. Early decompensation and higher mortality rates are also attributed to vitamin $D$ deficiency in these patients. ${ }^{21}$ Many studies have also suggested that adequate replacement of vitamin $D$ by using vitamin D supplements can improve the functional status, prognosis, CP score, model of end-stage liver disease score and overall morbidity of patients with liver disease. Therefore, it is recommended that this deficiency should be addressed promptly so that patients with chronic liver problems are benefited. ${ }^{22}$

\section{Conclusions}

Vitamin D deficiency is documented in the majority of patients afflicted by CLD, particularly those having advanced disease. As the disease advances, the levels become more deficient. Vitamin $D$ levels should be routinely checked in all patients suffering from advanced CLD, so that adequate replacement by vitamin $D$ supplements can be initiated as a therapeutic adjunct in managing such patients.

\section{Conflict of interest}

The authors have no conflict of interests related to this publication.

\section{Author contributions}

Conception of study objectives and/or design, drafting of the article and revising the article for important intellectual

Table 4. Univariate and multinomial regression analyses showing the different variables to predict vitamin D deficiency in the study group

\begin{tabular}{|c|c|c|c|c|c|}
\hline \multirow[b]{2}{*}{ Variable } & \multicolumn{2}{|l|}{ Univariate analysis } & \multicolumn{3}{|l|}{ Multinomial analysis } \\
\hline & OR $(95 \% \mathrm{CI})$ & $p^{*}$ & Chi-square value & OR $(95 \% \mathrm{CI})$ & $p^{*}$ \\
\hline Age & $3.72(3.26-4.18)$ & 0.000 & 70.48 & $1.26(1.15-1.38)$ & 0.000 \\
\hline Sex, female vs. male & $1.69(1.59-1.79)$ & 0.000 & 46.65 & - & 0.000 \\
\hline Etiology of liver disease & $1.50(0.57-2.43)$ & 0.523 & 0.738 & - & 0.947 \\
\hline MELD score & $2.69(2.35-3.03)$ & 0.000 & 27.40 & $1.32(1.14-1.52)$ & 0.000 \\
\hline CP score & $2.89(2.51-3.26)$ & 0.000 & 32.55 & $2.10(1.46-3.01)$ & 0.000 \\
\hline $\mathrm{CP}$ class & $1.28(1.10-1.47)$ & 0.000 & 43.17 & $0.02(0.002-0.18)$ & 0.001 \\
\hline
\end{tabular}

* Bold font indicates statistically significant values.

Abbreviations: CI, confidence interval; CP, Child-Pugh; MELD, model of end-stage liver disease; OR, odds ratio. 
content (ZJ, AAD, NY), collection of data ( $S A, A K)$, analysis of data (ZJ, SA), interpretation of findings (ZJ, AK).

\section{References}

[1] Ramos-Lopez O, Martinez-Lopez E, Roman S, Fierro NA, Panduro A. Genetic, metabolic and environmental factors involved in the development of liver cirrhosis in Mexico. World J Gastroenterol 2015;21:11552-11566. doi: 10. 3748/wjg.v21.i41.11552.

[2] Mokdad AA, Lopez AD, Shahraz S, Lozano R, Mokdad AH, Stanaway J, et al. Liver cirrhosis mortality in 187 countries between 1980 and 2010: a systematic analysis. BMC Med 2014;12:145. doi: 10.1186/s12916-014-0145-y.

[3] Sebastiani G, Gkouvatsos K, Pantopoulos K. Chronic hepatitis C and liver fibrosis. World J Gastroenterol 2014;20:11033-11053. doi: 10.3748/wjg. v20.i32.11033.

[4] Peng Y, Qi X, Guo X. Child-Pugh versus MELD score for the assessment of prognosis in liver cirrhosis: a systematic review and meta-analysis of observational studies. Medicine (Baltimore) 2016;95:e2877. doi: 10.1097/MD. 0000000000002877

[5] Kang W, Kim SU, Ahn SH. Non-invasive prediction of forthcoming cirrhosisrelated complications. World J Gastroenterol 2014;20:2613-2623. doi: 10. 3748/wjg.v20.i10.2613.

[6] Malham M, Jørgensen SP, Ott P, Agnholt J, Vilstrup H, Borre M, et al. Vitamin D deficiency in cirrhosis relates to liver dysfunction rather than aetiology. World J Gastroenterol 2011;17:922-925. doi: 10.3748/wjg.v17.i7.922.

[7] Gatta A, Verardo A, Di Pascoli M, Giannini S, Bolognesi M. Hepatic osteodystrophy. Clin Cases Miner Bone Metab 2014;11:185-191. doi: 10.11138/ $\mathrm{ccmbm} / 2014.11 .3 .185$.

[8] Nair S. Vitamin d deficiency and liver disease. Gastroenterol Hepatol (N Y) 2010;6:491-493.

[9] Cholongitas E, Theocharidou E, Goulis J, Tsochatzis E, Akriviadis E, Burroughs K. Review article: the extra-skeletal effects of vitamin $D$ in chronic hepatitis C infection. Aliment Pharmacol Ther 2012;35:634-646. doi: 10. 1111/j.1365-2036.2012.05000.x.

[10] Cantorna MT, Snyder L, Lin YD, Yang L. Vitamin D and 1,25(OH)2D regulation of T cells. Nutrients 2015;7:3011-3021. doi: 10.3390/nu7043011.

[11] Venu M, Martin E, Saeian K, Gawrieh S. High prevalence of vitamin A deficiency and vitamin $\mathrm{D}$ deficiency in patients evaluated for liver transplantation. Liver Transpl 2013;19:627-633. doi: 10.1002/lt.23646.

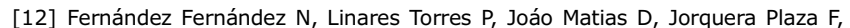
Olcoz Goñi JL. Vitamin D deficiency in chronic liver disease, clinical-epidemiological analysis and report after vitamin d supplementation. Gastroenterol Hepatol 2016;39:305-310. doi: 10.1016/j.gastrohep.2015.10.003.

[13] Dietrich CG, Götze O, Geier A. Molecular changes in hepatic metabolism and transport in cirrhosis and their functional importance. World J Gastroenterol 2016;22:72-88. doi: 10.3748/wjg.v22.i1.72.

[14] Jeong SW. Another face of contrast-enhanced ultrasonography: a diagnostic tool for cirrhosis. Gut Liver 2017;11:9-10. doi: 10.5009/gnl16563.

[15] Zhao XY, Li J, Wang JH, Habib S, Wei W, Sun SJ, et al. Vitamin D serum level is associated with Child-Pugh score and metabolic enzyme imbalances, but not viral load in chronic hepatitis B patients. Medicine (Baltimore) 2016;95: e3926. doi: 10.1097/MD.0000000000003926.

[16] Kumar R, Kumar P, Saxena KN, Mishra M, Mishra VK, Kumari A, et al. Vitamin D status in patients with cirrhosis of the liver and their relatives-A case control study from North India. Indian J Gastroenterol 2017;36:50-55. doi: 10.1007/ s12664-017-0727-7.

[17] Kiani IG, Shah F, Mansur SS. Frequency of severe vitamin-D deficiency in patients presenting to a tertiary care hospital in Islamabad. J Pak Med Assoc 2014;64:1138-1140

[18] Mehboobali N, Iqbal SP, Iqbal MP. High prevalence of vitamin D deficiency and insufficiency in a low income peri-urban community in Karachi. J Pak Med Assoc 2015;65:946-949.

[19] Konstantakis C, Tselekouni P, Kalafateli M, Triantos C. Vitamin D deficiency in patients with liver cirrhosis. Ann Gastroenterol 2016;29:297-306. doi: 10. 20524/aog.2016.0037.

[20] Junaid K, Rehman A, Jolliffe DA, Wood K, Martineau AR. High prevalence of vitamin $D$ deficiency among women of child-bearing age in Lahore Pakistan, associating with lack of sun exposure and illiteracy. BMC Womens Health 2015;15:83. doi: 10.1186/s12905-015-0242-x.

[21] Putz-Bankuti C, Pilz S, Stojakovic T, Scharnagl H, Pieber TR, Trauner M, et al. Association of 25-hydroxyvitamin $D$ levels with liver dysfunction and mortality in chronic liver disease. Liver Int 2012;32:845-851. doi: 10.1111/j.1478 3231.2011.02735.x.

[22] Chen EQ, Shi Y, Tang H. New insight of vitamin D in chronic liver diseases. Hepatobiliary Pancreat Dis Int 2014;13:580-585. doi: 10.1016/S1499-3872 (14)60295-2. 